\title{
Hard scattering and stochastic reformulation of parton energy loss
}

\author{
Tianyu Dai* \\ Department of Physics, Duke University \\ Durham, NC27708, USA \\ E-mail: tianyu.dai@duke.edu
}

\section{Steffen A. Bass}

Department of Physics, Duke University

Durham, NC27708, USA

E-mail: bass@phy . duke.edu

\section{Jean-François Paquet}

Department of Physics, Duke University

Durham, NC27708, USA

E-mail: jeanfrancois.paqueteduke.edu

\section{Derek Teaney}

Department of Physics \& Astronomy, Stony Brook University

Stony Brook, NY11764, USA

E-mail: derek.teaney@stonybrook. edu

\begin{abstract}
Hard partons lose energy when propagating through the quark-gluon plasma by interacting with plasma particles. These parton-plasma interactions consist of frequent soft interactions and occasional hard scatterings. A reformulated parton energy loss model building on a stochastic description of soft interactions was derived recently in the weakly-coupled limit. We numerically implement this reformulated model and apply it in the phenomenological regime. We validate this model by testing the dependence of the parton energy loss on the cutoff between soft and hard interactions, at both small and large coupling, for a static brick as well as a realistic hydrodynamic medium.
\end{abstract}

13th International Workshop on High-pT Physics in the RHIC/LHC era 19 - 22 March 2019

Knoxville, Tennessee, USA

${ }^{*}$ Speaker. 


\section{Introduction}

The weakly-coupled effective kinetic approach is a formalism to investigate the interactions between light partons and a weakly-coupled quark-gluon plasma. In this approach, the dynamics of partons propagating in the plasma is described by transport equations, and the parton-medium interactions are evaluated perturbatively [1]. A leading order implementation of this formalism is MARTINI [2], which formulates the parton-plasma interactions as $1 \rightarrow 2$ inelastic processes and $2 \rightarrow 2$ elastic processes. However, elastic and inelastic interactions can be further divided as hard and soft collisions. The larger number of soft collisions enables them to be described by a stochastic process. Rare hard scatterings are evaluated separately with emission rates. A systematic division between soft and hard interactions was derived in Ref. [3].

This hard-soft reformulation of the parton energy loss is expected to be more efficient, since all the dynamics of soft interactions are encoded in a few drag and diffusion coefficients. This reformulation is also flexible, because the stochastic description of soft interactions can be extended to the phenomenological regime.

We numerically implement this hard-soft reformulated energy loss model in both the perturbative and phenomenological limit. We analyze the dependence of the single parton energy distribution and nuclear modification factor on the scales separating soft and hard interactions to verify this reformulated model. Our results indicate that this parton energy reformulation can be applied to the phenomenological regime.

\section{Description of the Reformulated Model}

\subsection{Hard interactions}

For inelastic interactions, we denote the energy of the radiated particle to be $\omega$. The cutoff between soft and hard inelastic interactions is set to be $\mu_{\omega}$, with $\mu_{\omega} \lesssim T$. T is the temperature of the plasma. When $\omega<\mu_{\omega}$, the interaction is known as small- $\omega$ interaction, which is treated as a drag and diffusion process. When $\omega>\mu_{\omega}$, the interaction is considered as a large- $\omega$ interaction, which can be solved by emission rates calculated from AMY integral equations[4].

For elastic interactions, we define $\tilde{q}_{\perp} \equiv \sqrt{q^{2}-\omega^{2}}$. A similar cutoff $\mu_{\tilde{q}_{\perp}}$ is defined to separate soft and hard elastic interactions, with $g T \ll \mu_{\tilde{q}_{\perp}} \ll T, g=\sqrt{4 \pi \alpha_{s}}$, where $\alpha_{s}$ is the strong coupling constant. Small- $\tilde{q}_{\perp}$ interactions are described as a diffusion process, while large- $\tilde{q}_{\perp}$ interactions are determined by collision rates calculated from matrix elements. We use the vacuum matrix elements to treat large- $\tilde{q}_{\perp}$ interactions here, because at small coupling, the screening effects are encoded in the drag and diffusion coefficients. We also assume that the energy of the propagating hard parton $p$ is much larger than the temperature and energy transfer, so that only the t-channels of the matrix elements are included in the calculation.

\subsection{Soft interactions}

Drag and diffusion Since we only track partons with sufficiently high energy $(p>10 \mathrm{GeV})$, the number of particles is effectively preserved in both elastic and inelastic soft interactions. In this work, identity-preserving soft interactions, elastic and inelastic, are described stochastically with drag and diffusion. 
When the exchanged energy is small, the Boltzmann equation can be approximated as a Fokker-Planck equation:

$$
\frac{P \cdot \partial f(\vec{p})}{P \cdot u}=\frac{\partial}{\partial p^{i}}\left[\eta_{D}(p) p^{i} f(\vec{p})\right]+\frac{1}{2} \frac{\partial^{2}}{\partial p^{i} \partial p^{j}}\left\{\left[\hat{p}^{i} \hat{p}^{j} \hat{q}_{L}(p)+\frac{1}{2}\left(\delta^{i j}-\hat{p}^{i} \hat{p}^{j}\right) \hat{q}(p)\right] f(\vec{p})\right\}
$$

where $P=(p, \vec{p})$ is the four-momentum of the parton, $f(\vec{p})$ is the distribution function of the parton, $u$ is the four-velocity of the medium, $\eta_{D}$ is the drag, $\hat{q}_{L}$ and $\hat{q}$ are the longitudinal and transverse momentum diffusion coefficients. Elastic interactions contribute to both $\hat{q}_{L}$ and $\hat{q}$ [3], while inelastic interactions only contribute to $\hat{q}_{L}$. These diffusion coefficients are calculated perturbatively. The elastic transverse diffusion coefficient is

$$
\hat{q}=\frac{g^{2} C_{R} T m_{D}^{2}}{4 \pi} \ln \left[1+\left(\frac{\mu_{\tilde{q}_{\perp}}}{m_{D}}\right)^{2}\right]
$$

where $C_{R}$ is the Casimir factor, $m_{D} \equiv g T \sqrt{N_{c} / 3+N_{f} / 6}$ is the leading order Debye mass, $N_{c}$ and $N_{f}$ are the number of colors and flavors involved in the interactions. The elastic longitudinal diffusion coefficient is

$$
\hat{q}_{L}^{\text {elas }}=\frac{g^{2} C_{R} T M_{\infty}^{2}}{4 \pi} \ln \left[1+\left(\frac{\mu_{\tilde{q}_{\perp}}}{M_{\infty}}\right)^{2}\right]
$$

where $M_{\infty} \equiv \sqrt{m_{D}^{2} / 2}$ is the gluon asymptotic thermal mass. The inelastic longitudinal diffusion coefficients is

$$
\hat{q}_{L}^{\text {inel }}=\frac{(2-\ln 2) g^{4} C_{R} C_{A} T^{2} \mu_{\omega}}{4 \pi^{3}} .
$$

The drag coefficient $\eta_{D}$ is obtained by the Einstein relation to achieve detailed balance:

$$
\eta_{D}(p)=\frac{\hat{q}_{L}}{2 T p}+\frac{1}{2 p^{2}}\left(\hat{q}-2 \hat{q}_{L}\right)
$$

Conversion process Identity-nonpreserving soft interactions are known as conversion processes, in which the incoming parton changes its identity by exchanging a soft fermion with the medium. However, the conversion rates of both the elastic and inelastic conversion process are at the order of $T / p$. Since we conserve only the zeroth order term in $T / p$ in this work, we do not include conversion processes in our implementation.

\subsection{Reformulation}

The parton energy loss model is reformulated from elastic and inelastic interactions to large- $\omega$, large- $\tilde{q}_{\perp}$ interactions and a diffusion process:

$$
\mathscr{C}=\mathscr{C}^{2 \leftrightarrow 2}+\mathscr{C}^{1 \leftrightarrow 2}=\mathscr{C}^{\text {large }-\omega}\left(\mu_{\omega}\right)+\mathscr{C}^{\text {large }-\tilde{q}_{\perp}}\left(\mu_{q_{\perp}}\right)+\mathscr{C}^{\text {diff }}\left(\mu_{\omega}, \mu_{q_{\perp}}\right) .
$$

Treatments of different processes in the reformulated model are summarized in the Table 1. Each process of the reformulated model is dependent on the cutoff, but the cutoff should cancel out once we combine all the processes. In the following section, we verify the reformulated model by testing the cutoff dependence of the parton energy loss. 
Table 1: Treatments of reformulated energy loss

\begin{tabular}{|c|c|c|}
\hline \multirow{2}{*}{ soft interactions } & small- $\tilde{q}_{\perp}$ elastic & \multirow{2}{*}{ diffusion processes } \\
\cline { 2 - 3 } & small- $\omega$ inelastic & \\
\hline \multirow{2}{*}{ hard interactions } & large- $\tilde{q}_{\perp}$ elastic & vacuum matrix elements \\
\cline { 2 - 3 } & large- $\omega$ inelastic & AMY integral equations \\
\hline
\end{tabular}

\section{Numerical implementation}

We implement the reformulated energy loss model in the public version of the JETSCAPE framework [5]. JETSCAPE is a modular framework for simulating heavy ion collisions including both the production and propagation of hard probes. Each module can be replaced or modified independently.

We solve the Boltzmann transport equation using Monte Carlo methods. In our implementation, all the high energy partons $(p>10 \mathrm{GeV})$ generated in the events are tracked. At each time step, the position of the parton is updated, and then we determine whether a hard process can happen, and which hard process can happen by the total rate of the hard process.

For a chosen hard process, we sample $\omega$ or $\tilde{q}_{\perp}$ by differential collision rates. We evaluate the differential rate numerically and tabulate it for efficiency. With the sampled $\omega$ or $\tilde{q}_{\perp}$, the momentum of the parton is updated at each time step.

For a soft process, drag and diffusion describes the average effects of soft interactions on a parton, which should be considered at each time step. The Fokker-Planck equation describing the soft interactions (Eq. 2.1) is implemented stochastically as a Langevin equation, solved with the pre-point Ito scheme [6]:

$$
\begin{aligned}
& \frac{\Delta \vec{x}}{\Delta t}=\frac{\vec{p}}{E} \\
& \frac{\Delta \vec{p}}{\Delta t}=-\eta_{D} \vec{p}+\vec{\xi}(t)
\end{aligned}
$$

where $\vec{x}$ is the position, and $E$ is the energy of the parton. $\vec{\xi}$ is the thermal random force:

$$
\begin{aligned}
\left\langle\xi_{i}\right\rangle & =0 \\
\left\langle\xi_{i} \xi_{j}\right\rangle & =\frac{1}{\Delta t}\left[\hat{p}_{i} \hat{p}_{j} \hat{q}_{L}+\frac{1}{2}\left(\delta_{i j}-\hat{p}_{i} \hat{p}_{j}\right) \hat{q}\right]
\end{aligned}
$$

The momentum of the parton is updated using the drag coefficient $\eta_{D}$ and longitudinal diffusion coefficient $\hat{q}_{L}$ in the direction of motion, and is changed by transverse diffusion coefficients $\hat{q}$ in the perpendicular direction. The diffusion is driven by the thermal random force. The average energy loss resulting from the diffusion coefficients is zero, although the shape of the energy distribution is modified.

At the end of each time step, newly generated particles with sufficiently high energy are added to the list of active partons. 


\section{Results in a static medium}

\subsection{Inelastic energy loss}

In the reformulated parton energy loss model, hard and soft interactions are separated by a cutoff. The cutoff should satisfy the condition that interactions with energy scale smaller than the cutoff are frequent enough, in the sense that the diffusion process is still a good description. The drag and diffusion coefficients in this work are calculated using perturbation theory. It has been shown in Ref. [3] that the reformulated energy loss is equivalent to the original one in the small coupling limit. Thus, by choosing the cutoff in a proper range, the results calculated at small coupling limit should be independent on the cutoff. We first verify numerically that the energy loss is independent of the cutoff at small coupling, and repeat the same test at large coupling. Since inelastic and elastic interactions are treated independently in our implementation, we test the cutoff dependence for the inelastic cutoff $\mu_{\omega}$ and elastic cutoff $\mu_{\tilde{q}_{\perp}}$ separately.

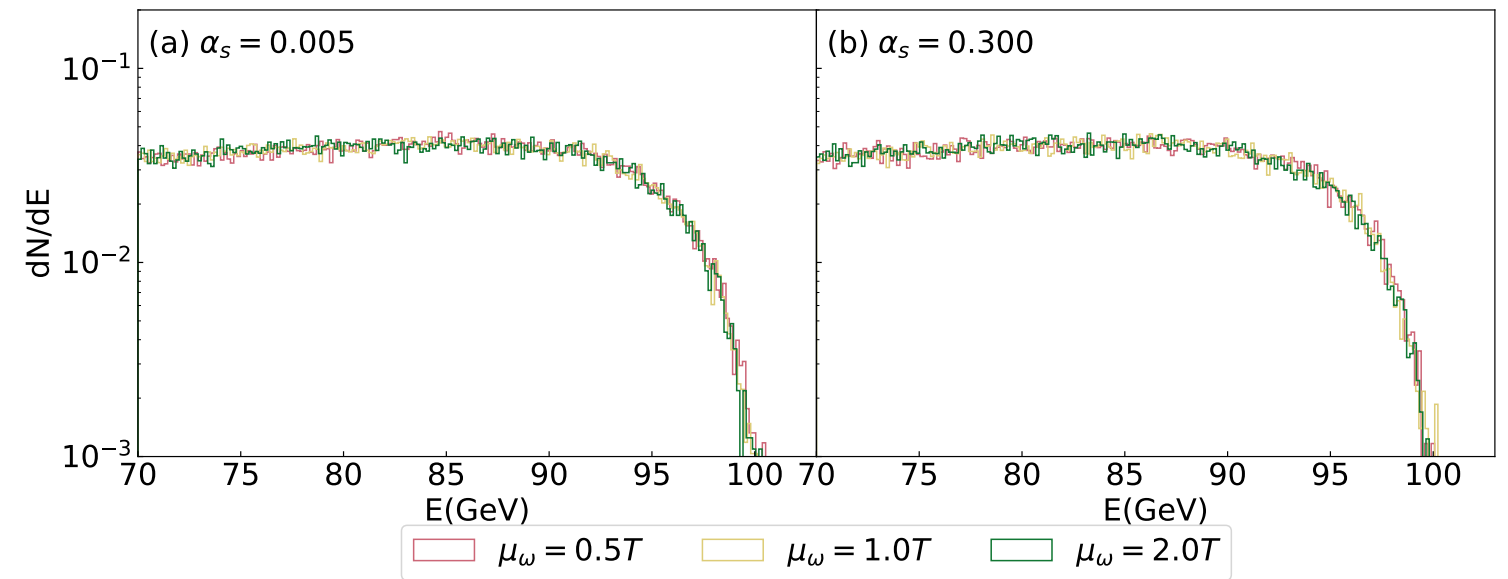

Figure 1: Energy distribution of a $100 \mathrm{GeV}$ gluon losing energy through inelastic processes in a static quarkgluon plasma at $\mathrm{T}=300 \mathrm{MeV}$ for different $\mu_{\omega}$. The propagation time is $\tau=3\left(0.3 / \alpha_{s}\right)^{2} \mathrm{fm} / \mathrm{c}$. The results are shown for two different coupling constants $\alpha_{s}$.

The energy distribution of a $100 \mathrm{GeV}$ gluon propagating through an infinite static medium with temperature $300 \mathrm{MeV}$ is shown in Figure 1, for different values of the cutoff $\mu_{\omega}$. We test at different coupling constants, and fix the propagation time as $\tau=3\left(0.3 / \alpha_{s}\right)^{2} \mathrm{fm} / \mathrm{c}$ to make the value of the energy loss similar for different coupling constants. Only inelastic energy loss is included in this test. At both small and large coupling, the energy distribution of the leading gluon is independent on the cutoff $\mu_{\omega}$.

We performed similar tests on quarks and partons with different initial energies. All of these tests demonstrated similar cutoff independence result, which indicates that this reformulation of inelastic interactions can be applied to phenomenological regime.

\subsection{Elastic energy loss}

The results of the cutoff dependence test for elastic interactions is shown in Figure 2. We calculate the energy distribution of a $100 \mathrm{GeV}$ gluon propagating in an infinite static medium 


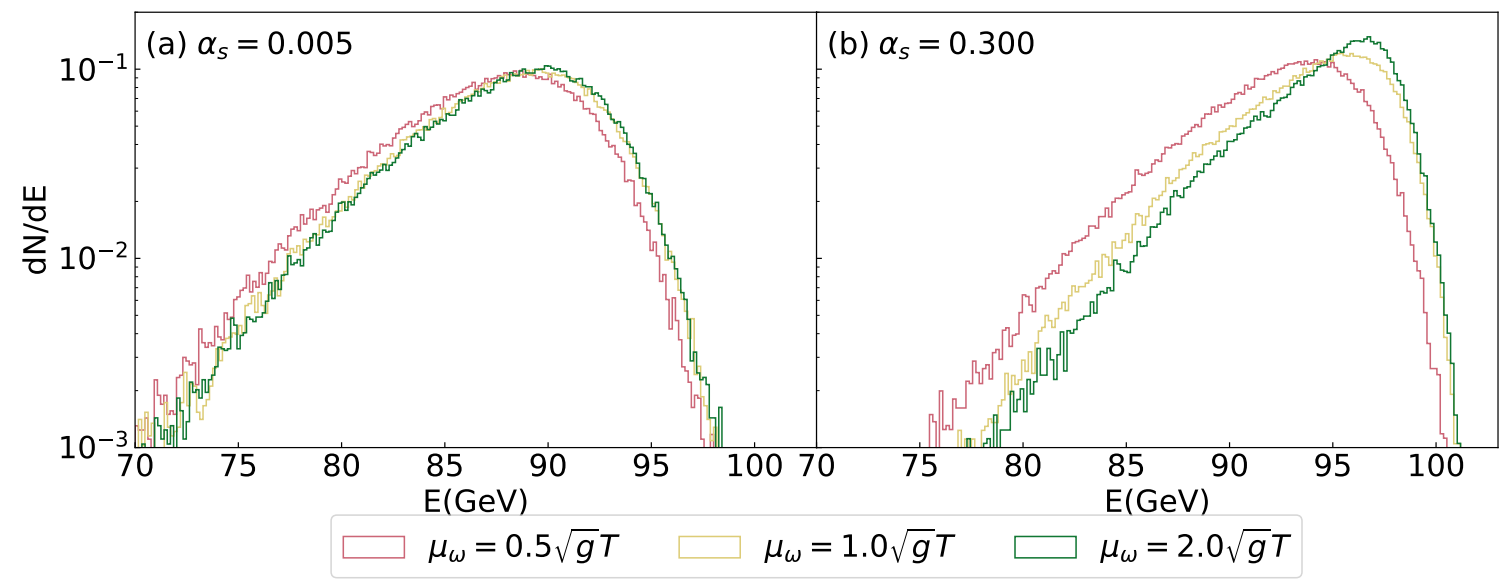

Figure 2: Energy distribution of a gluon with initial energy $E_{0}=100 \mathrm{GeV}$ losing energy through elastic processes in a static plasma with $T=300 \mathrm{MeV}$, for different elastic cutoff $\mu_{\tilde{q}_{\perp}}$. The propagation time is $\tau=3\left(0.3 / \alpha_{s}\right)^{2} \mathrm{fm} / \mathrm{c}$. The results are shown for two different coupling constants $\alpha_{s}$.

with temperature $300 \mathrm{MeV}$. We only allow elastic interactions between the leading gluon and the medium in this test. Similar to the previous test, we calculate with different elastic cutoff $\mu_{\tilde{q}_{\perp}}$ at different coupling constants.

The energy loss of elastic interactions has larger dependence on the cutoff than that of inelastic interactions. It is possible that this larger cutoff dependence is caused by the use of vacuum matrix elements in the treatment of large- $\tilde{q}_{\perp}$ interactions. At large coupling, the screening effect can affect interactions with energy scale larger than the cutoff. In Figure 2, the energy distribution has a smaller dependence on the cutoff at small coupling, which is consistent with this hypothesis.

\section{Results in a Real Hydrodynamic Medium}

The nuclear modification factor $R_{A A}$ is an important observable to quantify the parton energy loss. We test the dependence of $R_{A A}$ on the cutoff $\mu_{\omega}$ in a realistic hydrodynamic medium. In this test, both elastic and inelastic interactions are included. We use a single realistic hydrodynamic event, and assume that all partons are generated at the center of the medium. We set the coupling constant to $\alpha_{s}=0.3$. Parton jets are identified with the anti- $k_{T}$ algorithm ${ }^{1}$.

Test results with various inelastic collision cutoffs $\left(\mu_{\omega}=0.25 \mathrm{~T}, \mathrm{~T}\right.$ and $\left.2 \mathrm{~T}\right)$ are shown in Fig. (3). The figure shows that results with different $\mu_{\omega}$ are consistent within the statistical uncertainty.

\section{Summary \& outlook}

We implemented numerically the systematic reformulation of parton energy loss derived in Ref. [3]. The energy distribution of a hard parton propagating through a static medium is independent on the inelastic cutoff $\mu_{\omega}$ at both small and large coupling. For the elastic cutoff $\mu_{\tilde{q}_{\perp}}$, the

\footnotetext{
${ }^{1}$ For the anti- $k_{T}$ algorith, we use a jet radius of $R=0.4$. The rapidity range of the identified jets is $|y|<2.8$, the transverse momentum range of the jets is $p_{T}^{\text {jet }}>50 \mathrm{GeV}$, and the transverse momentum range of the partons in the jets is $p_{T}^{\text {parton }}>20 \mathrm{GeV}$.
} 


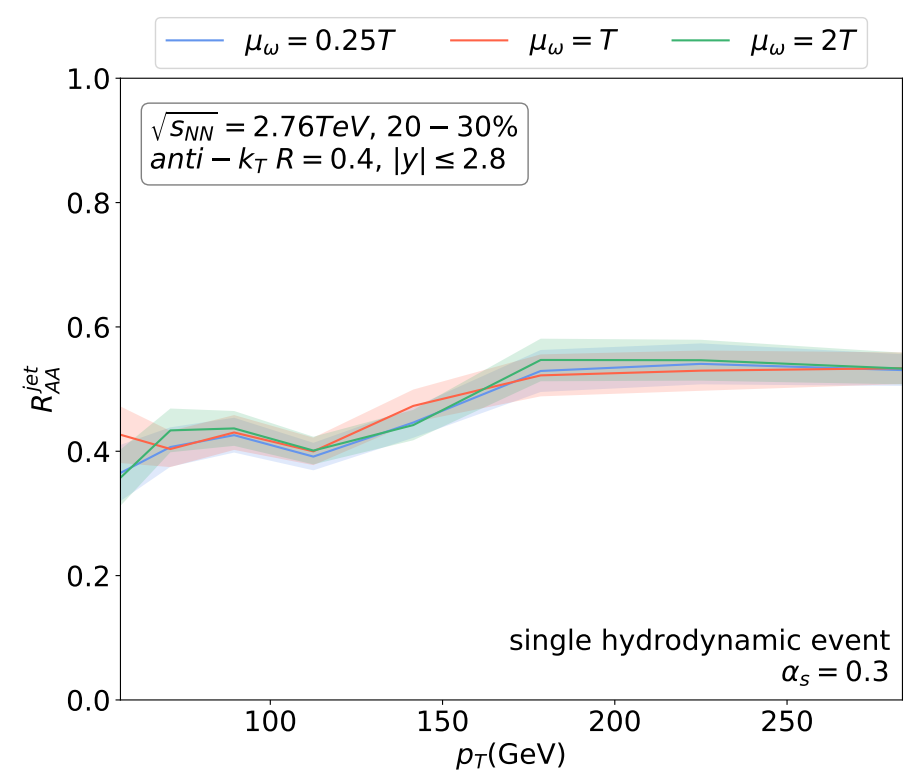

Figure 3: $R_{A A}$ of parton jets produced in a heavy ion collision with $\sqrt{s_{N N}}=2.76 \mathrm{TeV}$.

result exhibits a larger cutoff dependence at the large coupling, although cutoff independence is recovered at small coupling as expected.

The reformulated parton energy loss model presented in this work factorizes the collective effect of soft collisions into transport coefficients. The parameterizable nature of these drag and diffusion coefficients makes the reformulated energy loss model particularly suited for data-driven constraints. Additionally, this reformulated energy loss formalism makes possible the extension of the parton energy loss rates to next-to-leading order [3, 7]. More applications based on this reformulated parton energy loss model are expected in the future.

Acknowledgements We are grateful to Jacopo Ghiglieri, Weiyao Ke and Yingru Xu for their help with this project. We thank Bjoern Schenke and Heikki Mäntysaari for their collaboration in the early stage of this project, and the JETSCAPE Collaboration for their assistance with the JETSCAPE framework. This work was supported by the National Science Foundation under Award Number ACI-1550225 (T.D.), the U.S. Department of Energy under Award Numbers DE-FG0205ER41367 (S.A.B., T.D., J.-F.P.) and DE-FG02-88ER40388 (J.-F.P., D.T.).

\section{References}

[1] P. B. Arnold, G. D. Moore and L. G. Yaffe, Effective kinetic theory for high temperature gauge theories, Journal of High Energy Physics 2003 (2003) 030.

[2] B. Schenke, C. Gale and S. Jeon, MARTINI: An Event generator for relativistic heavy-ion collisions, Phys. Rev. C80 (2009) 054913 [0909.2037].

[3] J. Ghiglieri, G. D. Moore and D. Teaney, Jet-medium interactions at nlo in a weakly-coupled quark-gluon plasma, Journal of High Energy Physics 2016 (2016) 95.

[4] P. B. Arnold, G. D. Moore and L. G. Yaffe, Photon and gluon emission in relativistic plasmas, JHEP 06 (2002) 030 [hep-ph/0204343]. 
[5] J. Putschke, K. Kauder, E. Khalaj, A. Angerami, S. Bass, S. Cao et al., The jetscape framework, arXiv preprint arXiv:1903.07706 (2019) .

[6] F. Debbasch, K. Mallick and J. Rivet, Relativistic ornstein-uhlenbeck process, Journal of statistical physics 88 (1997) 945.

[7] S. Caron-Huot, $O(g)$ plasma effects in jet quenching, Physical Review D 79 (2009) 065039. 